\title{
Factores asociados a la baja calidad del esperma
}

\author{
Carlos Bryan Balitán Amoretty, Sabina Antea Blanco Knotek, \\ Alejandro Cabrera, Yeral Alexander Hernández. \\ Tutor: Dr. Clara González.Moncada / Asesor: Lic. Deybis Dinarte. \\ Departamento de Microbiología, Facultad de Ciencias Médicas, Universidad Nacional Autónoma de Nicaragua, \\ Managua, Nicaragua. \\ blancosabi@gmail.com, amoretty94@hotmail.com, yahh1993@live.com
}

\section{RESUMEN}

La calidad espermática en jóvenes está afectada por diversos factores. Este estudio es de carácter descriptivo, transversal. Con un universo de 412 alumnos de la carrera de Medicina de área Básica de la UNAN-Managua. La muestra, no probabilística por conveniencia, fue de 40 estudiantes entre 18-30 años. El instrumento que se aplicó fue un cuestionario con las variables de interés. El esperma se recolectó y analizó bajo parámetros de la OMS. El examen físico, químico y microscópico se reportaron alterados en 100\% de las muestras. El 100\% de las muestras presentaron alteraciones en el $\mathrm{pH}$ y $75 \%$ alteraciones microscópicas. $20 \%$ de los estudiantes refirieron hábitos tóxicos relacionándose con alteraciones en todos parámetros. El $75 \%$ se expuso a factores ambientales con reporte del examen físico seminal alterado en el $60 \%$, mientras que el químico en el $72.5 \%$ y el microscópico en el $60 \%$; de este un $10 \%$ se observó la morfología alterada, 12,5\% con motilidad menor a 70\%, vitalidad inferior a $80 \%$. En los estudiantes con dieta variada y ejercicio regular se reportó mejor calidad seminal en los parámetros evaluados. Los fármacos alteraron $40 \%$ de los parámetros evaluados. Los resultados de la investigación proporcionan datos epidemiológicos inexistentes en Nicaragua. Se recomienda cambiar el estilo de vida para mejorar la salud y vida sexual. Los factores asociados a la baja calidad seminal en los jóvenes de este estudio son prevenibles.

Palabras claves: Espermograma, Morfología, Motilidad, vitalidad.

\section{ABSTRACT}

In young sperm quality is affected by various factors. Performing a Descriptive, cross-sectionalstudy. Our universe was made up of 412 students of Basic Medicine degree course area of the Unan-Managua. Our sample, 40 students aged 18-30, not probabilistically selected for convenience completed a questionnaire; sperm parameters was analyzed under WHO.The physical, chemical and microscopic examinations were found altered in $100 \%$ of samples. All samples showed alterations in $75 \% \mathrm{PH}$ and microscopic alterations. $20 \%$ of students reported toxic habits, reporting changes in all parameters. $75 \%$ were exposed to environmental factors, physical examination $60 \%$ seminal altered, $72.5 \%$ and $60 \%$ chemical microscopic reported; This morphology $10 \%$, $12.5 \%$ with less than $70 \%$ motility, less than $80 \%$ vitality. Students with varied diet and regular exercise had better semen quality parameters evaluated. Drugs alter $40 \%$ of the evaluated parameters. Our research provides epidemiological data nonexistence in Nicaragua. We invite lifestyle change for better health and sexual life. Factors associated with low semen quality are perfectly preventable.

Keywords: Esperm, Morphology, Motility, Vitality 


\section{INTRODUCCIÓN}

El factor masculino en la infertilidad representa un 50\% en los aspectos etiológicos de la misma, indefinida, según estudios recientes, en diversos países se demuestra el deterioro progresivo de la concentración y calidad espermática. En los últimos años poco se ha avanzado en el conocimiento de la etiología y la fisiopatología reproductiva masculina, debido en parte al advenimiento de la técnica de inyección intracitoplasmática de espermatozoides, que ha facilitado el proceso reproductivo en una pareja aquejada de problemas de fertilidad. (Rodríguez, 2008)

En líneas generales, el hombre puede ver afectada su fertilidad por alteraciones mecánicas, en las que se dificulta el depósito de espermatozoides en el tracto femenino (epispadia, hipospadia, eyaculación precoz) o más frecuente por alteraciones de la concentración o calidad del esperma en el hombre (Rodríguez, 2008).

Las alteraciones en la concentración del semen detectadas podrían tener sus causas en el periodo embrionario, (es decir, originarse antes incluso del nacimiento de esos varones) y deberse en zonas altamente industrializadas a la exposición del embrión a los disruptores endocrinos a través del cordón umbilical, estas alteraciones se han encontrado en jóvenes y hombres mayores en zonas con mayor índice de industrialización. (Asociación Española de Andrología (ASESA) y ANACER (Asociación Nacional de Clínicas de Reproducción), 2008).

Investigadores de la Universidad de Rosario en Argentina plantean que el tabaquismo, un "hábito de vida" con una alta prevalencia entre los jóvenes, compromete la concentración y la morfología espermática, asociándose con un mayor número de células espermáticas inmaduras. Se ha supuesto que algunos de los componentes del cigarrillo se asocian a la disminución de gonadotrofinas liberadas por la pituitaria, esta estimula el desarrollo de los testículos. Se ha comprobado que la exposición al aire contaminado con hidrocarburos aromáticos policíclicos, se asocia también a la disminución de hormonas necesarias en la espermatogénesis, y se ha analizado su efecto en pacientes con infertilidad idiopática, que afecta generalmente al 39\% de los hombres. Factores tan comunes como el sedentarismo afecta varios parámetros del espermograma, al igual que una dieta pobre en antioxidantes actúa negativamente sobre el ADN espermático, sumado a un déficit de micronutrientes afecta a los espermatozoides, siendo estos esenciales en la formación de los mismos (Rodríguez, 2008).

Como consecuencia de lo expuesto, el propósito de esta investigación es la de presentar los resultados de una primera fase de un trabajo de investigación más amplio. El objetivo de esta primera 
fase fue determinar la calidad del semen de jóvenes universitarios según los criterios de la OMS. Para poder conocer algunos factores asociados a los cambios en los parámetro del espermograma. En la siguiente fase de esta investigación se harán intervenciones de información y educación así como brindar suplementos de hierro y ácido fólico para estudiar el efecto en los parámetros del espermograma de jóvenes que se les reportó con alteración.

A nivel mundial existe gran preocupación por la disminución de la calidad del semen en varones de países industrializados, se hace necesario ampliar el presente estudio ya que en Nicaragua todavía no hay estudios nacionales que reporten la calidad del semen en jóvenes nicaragüenses y cada vez, existen más parejas con problemas de fertilidad, es necesario conocer la calidad del semen de nuestros jóvenes y como se ven afectados por factores externos y ambientales (ANACER, 2002).

\section{MATERIAL Y MÉTODOS}

Se trata de un estudio descriptivo de corte transversal realizado en el mes de Agosto del 2014 en la Facultad de Ciencias Médicas de la UNAN, Managua, Nicaragua. El universo estuvo conformado por estudiantes de Medicina de áreas básicas del sexo masculino de la Facultad de Ciencias Médicas, UNAN-Managua conformado por 412 alumnos.

La muestra constituida por 40 alumnos fue tomada no probabilísticamente por conveniencia y representa un $9,7 \%$ del universo.

Como criterios de inclusión, se consideró a todo estudiante activo entre 18-30 años, con más de 2 días de abstinencia y cuya muestra fue tomada por masturbación directa en un frasco estéril proporcionado por el laboratorio. Se excluyó a aquellos que tomaron la muestra de coito o con condón y a alumnos con alguna patología que afecte el espermograma. Una vez cumplidos los criterios de inclusión los 40 participantes llenaron cuestionarios previo consentimiento informado.

Las fuentes de información fueron primarias por medio de los datos recogidos de la encuesta y secundaria a través de los informes de laboratorio.

\section{Recolección de la muestra}

Se les entregó el vaso estéril de boca ancha, se rotuló con el código asignado al participante, hora de recolección y fecha. Se les acondicionó un local cercano al laboratorio para la recolección del semen por el método de la masturbación directa, previa higiene de los genitales y las manos. Una vez recolectada la muestra el recipiente fue tapado de forma hermética, se guardó en una bolsa de papel 
para respetar la intimidad de los participantes y se llevó inmediatamente al laboratorio de Microbiología y Parasitología de la Facultad de Medicina donde la muestra fue analizada.

La muestra se analizó en un local con temperatura ambiente para evitar alteraciones en la lectura siguiendo la guía de la OMS, 1999. Se realizó un examen macroscópico y otro examen microscópico de la muestra de semen:

Evaluación inicial macroscópica siguió las recomendaciones del Manual de la OMS el cual indica que el análisis debe realizarse antes de transcurridos 30 minutos desde la recogida de la muestra. Además, la evaluación del semen se realiza con los aspectos macroscópicos del mismo.

\section{Análisis Físico}

Licuefacción: Por observación directa a los 15 minutos de recolectada la muestra. Se consideró normal cuando la muestra se observó licuada, homogénea, sin grumos ni coágulos antes de los 60 minutos.

Aspecto o apariencia: Se observó en forma directa en el recipiente el aspecto de esta y se anotaba posteriormente el resultado. Se consideró normal al observar el semen homogéneo, gris opalescente.

Viscosidad o consistencia: La viscosidad o consistencia del semen se evaluó aspirando la muestra en una pipeta permitiendo la caída libre de las gotas para observar la longitud del filamento que se forma. Una muestra normal deja caer gotas pequeñas y bien definidas o un filamento no mayor de $2 \mathrm{~cm}$.

Volumen: Para medir el volumen se utilizó un cilindro graduado de base cónica en el cual se vertió el semen proveniente del frasco donde el participante depositó la muestra. Luego se anotó el volumen en mililitros (ml). El manual establece el límite inferior de referencia en $2 \mathrm{ml}$.

\section{Examen químico}

PH: El análisis se realiza en la primera hora de recolección por medio de una cinta reactiva de orina, después de exponer ésta a la muestra, se lee a los 15 minutos. El límite inferior de referencia establecido es de 7,2. Densidad: Para medir este parámetro se utilizó cinta reactiva de orina que se sumergió en la muestra esto provocó el cambio de color de la cinta y este se compraba con los colores que indicaban el valor determinado de densidad. Una vez comparado el color se le asignaba un valor de referencia que no debe ser inferior a 1,020. 


\section{Análisis Microscópico}

Se utilizó un microscopio óptico de luz blanca.

Motilidad: La motilidad de los espermatozoides se evaluó en una muestra de $10 \mu \mathrm{L}$ entre lámina y laminilla, por duplicado. Se observó de manera directa al microscopio y se clasificó la motilidad según los criterios de la OMS 1999.

Vitalidad Espermática (Test de Eosina): Este parámetro se utilizó con el fin de averiguar cuantos espermatozoides inmóviles están vivos o muertos. El porcentaje de espermatozoides vivos se determinó por la implementación del test de eosina.

Se mezcló una gota $(10 \mu \mathrm{L}$ a $15 \mu \mathrm{L})$ del semen con una gota del colorante con eosina al $0,5 \%$ en una lámina portaobjeto y se cubrió con una laminilla para dejarla reposando durante unos 30 segundos y se procedió a contar 100 espermatozoides (coloreados y no coloreados), con una magnificación de 100X. Los espermatozoides vivos conservaron su membrana intacta porque no absorbieron el colorante, en tanto que los muertos adquirieron la coloración. El resultado se expresó en porcentaje que posteriormente fue anotado con los demás datos obtenidos. Según la OMS de 1999 los valores deben ser no menores de $75 \%$ espermatozoides vivos.

Recuento: Para el recuento de espermatozoides se utilizó la cámara de Neubauer. Se contó en el cuadrante central que se utiliza para el recuento de los glóbulos rojos. Con base en lo estimado en la observación inicial de la muestra, se diluyó el semen con solución fisiológica, se llenaron ambos lados de la cámara de Neubauer con $10 \mu \mathrm{L}$ de la dilución utilizando pipetas automáticas y se procedió a contar con una magnificación de 100X.

Para las muestras que contienen menos de 3 espermatozoides en el cuadro pequeño, se debió contar todos los cuadrados (que contiene 25 cuadrados pequeños se leía de izquierda a derecha). Si hay más de 3 espermatozoides se contó solo 5 cuadros pequeños de los extremos y uno en el centro. Los resultados de ambos lados de la cámara se promediaron y el valor se dividió por el factor de conversión; el resultado final corresponde al número (en millones) de espermatozoides por ml de eyaculado o concentración de los espermatozoides por $\mathrm{mL}^{3}$. En el recuento solo se incluyó espermatozoides completos con cabeza y cola que estén estrictamente dentro del cuadro. Los defectuosos se toman en cuenta sólo que tengan cabeza o cola, se deben contar aparte e informar en el resultado. El recuento debe ser mayor de 20 millones de espermatozoides por $\mathrm{ml}$ de eyaculado según la OMS 1999. 
Morfología Espermática: La evaluación de la morfología de los espermatozoides se realizó con coloración Cristal Violeta. Se utilizó un objetivo ocular de 10X y un objetivo de inmersión de 100X, seleccionando varias áreas sistemáticamente sin mucho colorante porque esto dificultaba la lectura correcta de la muestra. El espermatozoide debió tener la cabeza, el segmento intermedio y la cola normal para considerarse normal. Si un espermatozoide tenía más de un defecto, se informó con el defecto más importante en este orden: cabeza, segmento intermedio, cola. El resultado se expresó en porcentaje, después de contar 100 espermatozoides. Las formas normales presentes debe ser por lo menos el 15\% de espermatozoides sin alteraciones morfológicas.

Elementos Agregados: En las muestras coloreadas también se identificó la presencia de otros tipos de células como son los leucocitos, las formas inmaduras de los espermatozoides, las células epiteliales del tracto uretral y de la próstata, y microorganismos. El hallazgo de estos debe ser menor de $1 \times 10^{6} / \mathrm{ml}$.

Aglutinación: La aglutinación de los espermatozoides puede ocurrir cabeza con cabeza, segmento intermedio con segmento intermedio, cola con cola o puede ser mixta, por ejemplo cabeza con cola. La aglutinación es sugestiva de presencia de anticuerpos anti espermatozoides y se debe evaluar al momento de determinar la motilidad de los espermatozoides. Se puede informar semicuantitativamente en escala de cruces.

\section{Procesamiento y presentación de resultados.}

Los datos recolectados fueron ingresados y procesados en una base de dato de EpiInfo 7.1.4. Los resultados obtenidos se presentan en tablas y gráficos. (CDC, 2014)

El análisis se presenta en tablas de frecuencia simple y relativa, y medidas de tendencia central para las variables cuantitativas.

\section{RESULTADOS}

En cuanto a los parámetros del espermograma evaluados por la OMS el 82.5\% presentó alteraciones en al menos uno de los parámetros del examen físico, principalmente la viscosidad, aumentada en el $57.5 \%$ y disminuida en el $37.5 \%$.

El examen químico $100 \%$ afectado; $\mathrm{PH}$ en el $20 \%$ y densidad del $92.5 \%$. El examen microscópico presentó alteraciones en 100\% de las muestras: motilidad entre $70-80 \%$ en el 65\% de las 
muestras. En relación a la vitalidad se reportó que 72.5\% tiene vitalidad del 80-90. El 87.5\% tiene entre $90-99 \%$ de morfología, esto es relativamente normal.

El recuento espermático se vio alterado en el $37.5 \%$, de estos hasta el $15 \%$ presentó oligozoospermia. El conteo en el esperma de "otros elementos celulares" evidenció en un 97.5\% de las muestras leucocitos, $70 \%$ eritrocitos y $25 \%$ bacterias.

La morfología resulta preponderante en un espermograma, ya que es uno de los principales determinantes de sub-fertilidad e infertilidad.

Tabla 1. Principales anomalías morfológicas

\begin{tabular}{ccc}
\hline Anormalidades de la Cabeza & Frecuencia & Porcentaje \\
\hline Cabeza Amorfa & $18 / 40$ & $45 \%$ \\
Cabeza Grande y Amorfa & $8 / 40$ & $20 \%$ \\
Cabeza Doble y Vacuolada & $10 / 40$ & $25 \%$ \\
Cabeza Piriforme y grande & $8 / 40$ & $20 \%$ \\
Anormalidades de Cola & Fr & $\%$ \\
TC: cola larga & $10 / 40$ & $25 \%$ \\
TC: Cola Enrollada & $9 / 40$ & $22.5 \%$ \\
TM: Cola Múltiple & $5 / 40$ & $12.5 \%$ \\
Oglutinación & Fr & $12.5 \%$ \\
\hline Otras & $5 / 40$ & $\%$ \\
\hline
\end{tabular}

La principal anormalidad de cabeza con un $45 \%$ fueron las cabezas Amorfas; En cuanto a las alteraciones de la cola las más frecuente con 25\% fue cola larga. Finalmente entre las alteraciones evidenciadas y clasificadas como "otras" se describe la ocurrencia de las aglutinaciones en un12.5\%.

La proporción entre los que fuman y consumen alcohol es de $45 \%$, de estos $35 \%$ al examen físico, 20\% al Examen Químico 30\% al examen microscópico presentaron alteraciones. 
El 50\% refirió usar fármacos, hasta un 12\% antibióticos; así 47.5\% presentó alteraciones en el examen físico; el 50\% del examen químico y 40\% daño microscópico.

El 97.5\% de la población en estudio hace uso de equipos electrónicos, ya sean celulares o laptops sobre o entre las piernas, representando esto un importante factor que altera los parámetros del espermograma como lo refleja la tabla $\mathrm{N}^{\circ} 2$.

\section{Factores que alteran el espermograma}

El 77.5\% de la muestra tienen una alimentación variada que consta de productos animales, vegetales y minerales, de estos $60 \%$ presentó alteración en el examen físico, $100 \%$ en el examen químico y un $55.5 \%$ en el examen microscópico. En caso de los que tienen una dieta estricta a base de productos animales y minerales representan un 15\% de la muestra todos presentaron resultados anormales en los exámenes físico, químico y microscópico. Los que consumen productos animales y vegetales representan un 5\% de la muestra estos en su totalidad presentaron alteraciones en los exámenes físico, químico mientras que el examen microscópico todos fueron normales. Los participantes con dieta a base de minerales y vegetales obtuvieron resultados anormales en todos los exámenes ya antes mencionados.

Tabla 2. Porcentaje de alteraciones y principales factores asociados

\begin{tabular}{ccccc}
\hline & Alcohol y Cigarrillos & Factores Químicos & $\begin{array}{c}\text { Factores } \\
\text { ambientales }\end{array}$ & $\begin{array}{c}\text { Dispositivos } \\
\text { electrónicos }\end{array}$ \\
\hline PH & $\%$ & $\%$ & $\%$ & $\%$ \\
\hline Densidad & $7.5 \%$ & $15 \%$ & $17.5 \%$ & $17.5 \%$ \\
Volumen & $17.5 \%$ & $42.5 \%$ & $70 \%$ & $85 \%$ \\
Concentración & $17.5 \%$ & $17.5 \%$ & $30 \%$ & $37.5 \%$ \\
Motilidad & - & $12.5 \%$ & $22.5 \%$ & $27.5 \%$ \\
Vitalidad & - & $12.5 \%$ & $12.5 \%$ & $10 \%$ \\
Morfología & - & $7.5 \%$ & $15 \%$ & $15 \%$ \\
\hline
\end{tabular}

El 80\% realiza ejercicio de estos $57.5 \%$ tiene alteraciones en el examen microscópico; del $20 \%$ que no realizan ejercicio todos presentaron alteraciones en el examen químico y $15 \%$ alteraciones al microscopio. 
El 30\% de los participantes consume vitaminas; en el 70\% restante, el examen físico el $62.5 \%$ esta alterado. En el químico el 100\% es anormal y las alteraciones microscópicas representan el $52.5 \%$.

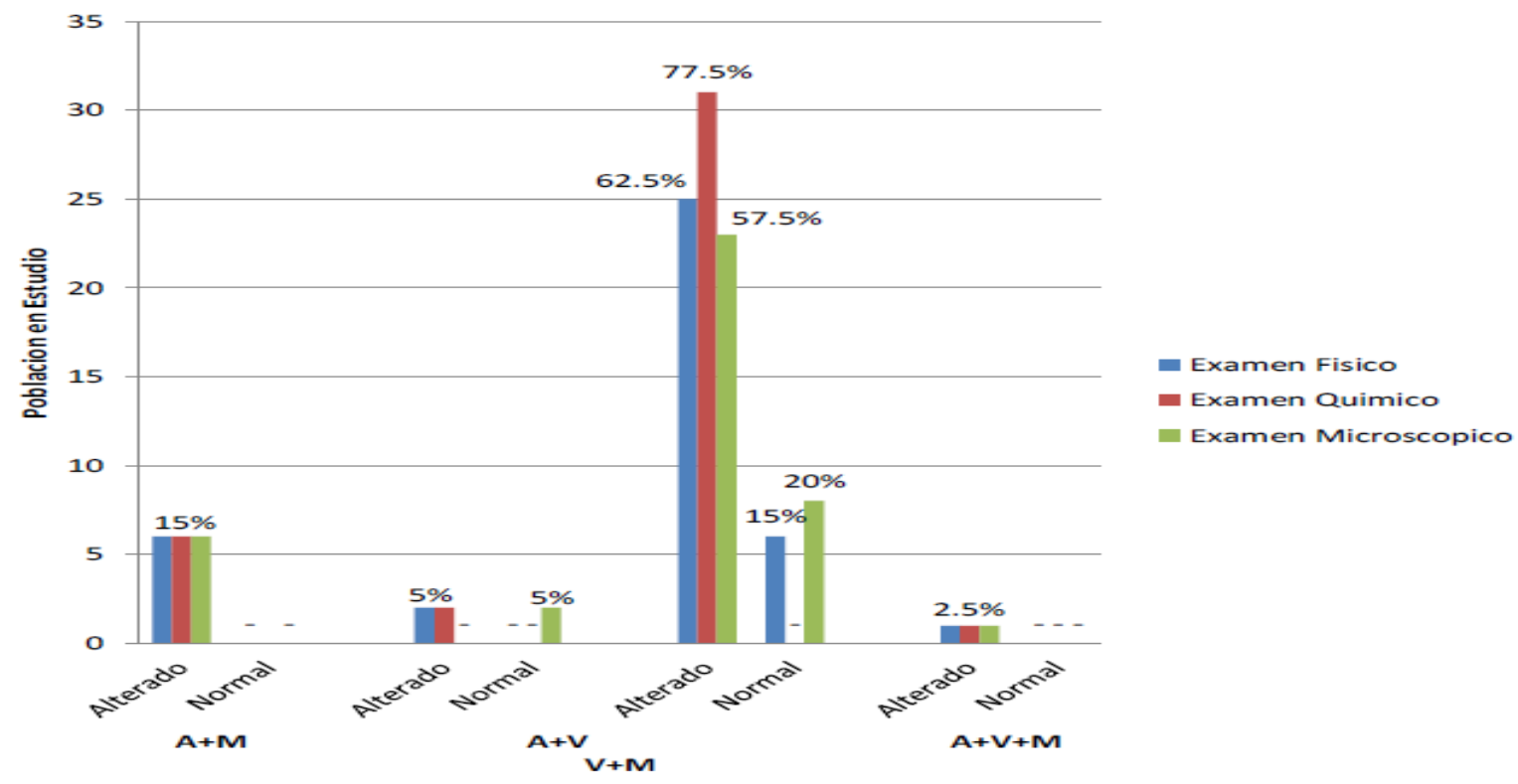

Figura 1: Alimentación y su relación con los parámetros evaluados en el espermograma.

\section{Aspectos sociodemográficos y espermograma}

Se encontró que más del 50\% de los estudiantes habían iniciado su vida sexual, 35\% tenía una vida sexual de riesgo al tener relaciones ocasionales sin uso de preservativo. Sin embargo, al realizar un análisis no se evidenció, según los datos obtenidos, que este hecho tuviera relación con alguna alteración, sea esta grave, en los parámetros del espermograma que se podrían traducir en sub-fertilidad e incluso infertilidad, a no ser que la conducta de riesgo conllevara a sufrir una infección de transmisión sexual por ejemplo (Merino Ruz MC, 2007). El 82.5\% habitualmente practicaba la masturbación, resultando que esta práctica mejora significativamente los parámetros del espermograma, principalmente el recuento (Jeyendran R, 1984).

\section{Espermograma}

A nivel fisiopatológico están demostrados los diversos mecanismos que conllevan al daño espermático por la exposición a diversos factores siendo estos por ejemplo el alcohol y los cigarrillos, evidenciándose que alteraban los valores normales de todos los parámetros del espermograma, siendo 
responsables de la producción de especies reactivas de oxígeno y afectando los niveles de gonadotrofinas produciéndose células inmaduras y dañadas a nivel morfológico (AdelZalata, 1995).

El recuento espermático se vio alterado en un cuarto de las muestras, llegando a ser $<10$ millones en la mitad de estos (12\%), y se evidenció que dos de estas muestras tenían poliespermia con más de 160 millones de células espermáticas.

Se realizó el conteo de otros elementos celulares a la hora de evaluar el esperma y se encontró en un 97.5\% de las muestras la presencia de leucocitos en cantidad variable, en un $70 \%$ la presencia de eritrocitos y en un $25 \%$ bacterias, todo esto asociado a traumas, exposición a factores ambientales, químicos, entre otros, causando daño a nivel hormonal disminuyéndose el umbral del estímulo necesario para la producción celular sexual en números adecuado (Sigman, 2009).

Un análisis de las principales anormalidades morfológicas evidencio que más del 50\% de estas fueron macrocéfalos, en las alteraciones de la cola la alteración más frecuente con $16.4 \%$ fue la cola larga, se describen en un 8.2\% las aglutinaciones. Todas estas son alteraciones microscópicas que se pueden atribuir a factores ambientales, sin embargo el daño microscópico se atribuye a infecciones a los que las células sexuales son muy lábiles, incluso el aumento de la temperatura regional causa el daño a este nivel, las aglutinaciones pueden responder a la presencia de reacciones con anticuerpos presentes a nivel testicular.

\section{Factores asociados a la baja calidad espermática.}

El 100\% de los participantes consume cafeína y hasta 40\% lo hace más de una vez al día, se ha demostrado que la cafeína afecta la concepción. Cuánto más café bebas, mayores son las posibilidades de reducir el número de espermatozoides, su movilidad y morfología. Las investigaciones científicas han notado un incremento en el número de abortos espontáneos en parejas cuyo padre bebía gran cantidad de café.

La exposición a químicos (pesticidas, pintura de casa, aerosoles) tienen repercusiones sobre las hormonas que regulan la función reproductiva y se ha analizado su efecto en pacientes con infertilidad idiopática, que afecta generalmente al 39\% de los hombres Hernández 2008). El 45\% de nuestra muestra estuvo expuesta a diferentes químicos, afectando en más del $30 \%$ los parámetros químicos y microscópicos. 
Los equipos electrónicos juegan un papel importante en la producción de radiación electromagnética la cual es culpable de que los espermatozoides tengan déficit en motilidad y conteo Espermático.

\section{Factores asociados a la mejoría de la calidad espermática}

Los participantes que tenían el hábito de ejercitarse presentaron alteraciones, sin embargo no tan significativas como la de aquellos quienes eran más sedentarios. Un equipo dirigido por la investigadora Diana Vaamonde estudió las diferencias en los perfiles hormonales y seminológicos entre hombres físicamente activos y sedentarios. Los resultados revelaron que los sujetos físicamente activos son los que muestran los mejores valores seminales, mejor morfología y mejor velocidad progresiva total.

La deficiencia de zinc puede producir una disminución en el volumen del semen y la testosterona. Esto se evita consumiendo alimentos ricos en zinc como ostras, mariscos, carnes, semillas de calabazas. No puede faltar el ácido fólico que es primordial, este se encuentra en legumbres, vegetales de hoja verde y frutos secos, por ende se demuestra que los participantes que tenían una alimentación balanceada tuvieron mejores resultados en sus parámetros. Esto pone en evidencia que una alimentación completa y balanceada con minerales, vegetales y productos animales ayuda a la mejoría significativa de todos los parámetros en estudio.

\section{Alternativa terapéutica}

Estudio realizado en Caracas Venezuela por Rodríguez (2008) en el Servicio de fertilidad. Maternidad "Concepción Palacios" en el 2008, sobre el efecto del ácido fólico (5mg) y zinc (50 mg) como tratamiento en pacientes masculinos subfértiles, con diagnóstico de astenospermia, oligospermia y teratorpermia por un periodo de 90 días, o con pacientes con alteración de la motilidad, concentración o morfología espermática, quedó demostrado que la motilidad aumenta de (44.37\% a 55.2\%), las formas espermáticas normales pasaron de $54.1 \%$ a $55.6 \%$ y las formas anormales disminuyeron de $44,29 \%$ a $44,25 \%$.

De tal forma se concluyó que el tratamiento empleado mejora significativamente la motilidad y la morfología espermática en el hombre sub-fértiles (Rodríguez, 2008). 


\section{CONCLUSIONES}

Las características sociodemográficas y de sexualidad en los participantes recogidas en la información de los cuestionarios compilaron datos interesantes como la conducta sexual de riesgo tomando en cuenta que la mayoría de los encuestados eran jóvenes entre 19 y 30 años, sin embargo no se demostró relación con las alteraciones en los parámetros del espermograma.

Todas las muestras en estudio presentaron alteraciones que en su mayoría afectaban el examen químico, así mismo los parámetros microscópicos resultaron afectados, y según la OMS 1999 son los principales parámetros al inferir sobre si existe sub-fertilidad e infertilidad.

Según los diversos análisis realizados a las muestras y su relación con la exposición a diversos factores que resultan nocivos directa o indirectamente para los espermatozoides, se obtuvo que los principales factores fueron entre otros el consumo de cigarrillos y la ingesta de alcohol, el uso de dispositivos electrónicos como celulares y laptops, la exposición a factores químicos y ambientales como las altas temperaturas, humedad entre otros.

Así pues se recomienda un cambio en el estilo de vida, evitar la exposición a los factores mencionados anteriormente e implementar medidas que impliquen factores asociados a la mejoría de la calidad espermática como una alimentación balanceada a base alimentos animales, minerales, vegetales; así mismo el uso de suplementos vitamínicos como ácido fólico y zinc han demostrado favorecer la motilidad y vitalidad espermática principalmente, finalmente en este estudio demostramos en los resultados que el ejercicio moderado favorece la mejoría de todos los parámetros del espermograma.

\section{AGRADECIMIENTOS}

A la Doctora Clara González Moncada por la tutoría brindada a lo largo de esta investigación; Al personal de laboratorio de Microbiología en especial al Licenciado Deyvi Dinarte por su asistencia técnica en la lectura de las muestras. 


\section{REFERENCIAS}

AdelZalata, TarekHafez, Frank Comhaire (1995). Evaluation of the role of reactive oxygen species in male infertility. Hum. Reprod. 10(6),44-51.

Auger J, Kunstsmann JM, Czyglik F, Jounannet P. (1995). Decline in semen quality among fertile men in Paris during the past 20 year. N Engl J Med. 332-281.

Calsen E, Giwwercman A, Keiding N, Skakkeback NE. (1992). Evidence of decreasing quality of semen during past 50 year. Br Med J. 305:609.

Comhaire F, Vermeulen L. (2003) Human semen analysis. Hum Reprod update. 1: 343-362

Eliasson R, Jhonsen O, Lindholmer C. Effect of zinc on human sperm respiration. Lif. Sci 1971; 10:1137-20.

Harrison Bashin S, Jameson L. (2003) Trastornos de los testículos y del sistema reproductor masculino; Principios de medicina interna de Harrison. 16ed. New york: Mc Graw-Hill. P. 11504-11565

Huaaya L, Sosa A, Delgado NM, Rosado A. A kinetic study of the participation of zinc in human spermatozoa S. RODRÍGUEZ, ET AL Rev ObstetGinecolVenez 180 metabolism, Lif Sci. 1973;13:1383-1394.

Jeyendran R, Van Der Ven H, Pérez-Peláez M, Crabo BG, Zaneveld L. (1984). Development of ram assay to assess the functional integrity of the human sperm membrane and its relationship to other semen characteristics. J. Reprod. Fert. 70:219-28.

Manual de laboratorio de la OMS para el examen del semen y de la interacción del semen y el moco cervical. (1992) 3ra ed. México: editorial médica panamericana.

Merino Ruz MC, De León Cervantes MC, García Flores CF. (2007) Male sterility and its association with genital disease and enviromental factor. GinecolobstetMex; 63: 427-431

Poirot C, Cherruau B. (2005) Infertilidad masculina: Aspectos clínicos e investigaciones biológicas. Acta BioquímClinLatinoam. 39:225-241.

Rodríguez Sh., Giustiniano B.,Abache E., Hurtado F. (2008) Tratamiento con ácido fólico y zinc en hombres subfertiles. Servicio de Fertilidad. Maternidad "Concepción Palacios "Maternidad “Concepción Palacios". RevObstetGinecolVenez. 6 
Sandstead HH, Prasad As, Schulert AR, Farid Z, MialeAJr, Bassilly S, et al. (1967) Human zinc deficiency, endocrine manifestations and response to treatment. Am J ClinNutr. 20:422-442.

Sigman M, Zinni A. (2009) Semen analysis an sperm function assays: what do they mean? Semen reprod med. 27: 115-123

World Health Organization. (1999) WHO laboratory manual for the examination of human semen and sperm cervical-mucus interaction.Cambridge : Cambridge University Press. 